Running Head: RAPID GUESSING CORRELATES IN PISA

\title{
An Investigation of Item, Examinee, and Country Correlates of Rapid Guessing in PISA
}

\author{
Joseph A. Rios ${ }^{\mathrm{a}} \&$ James Soland $\mathrm{d}^{\mathrm{b}, \mathrm{c}}$ \\ ${ }^{a}$ University of Minnesota \\ bUniversity of Virginia \\ 'NWEA
}

Author's Note

Joseph A. Rios: https://orcid.org/0000-0002-1004-9946

The first author holds departmental affiliation at the Department of Educational Psychology, University of Minnesota, Twin Cities. The second author holds departmental affiliation at the Department of Leadership, Foundations \& Policy, University of Virginia, and is also an affiliated research fellow at NWEA.

The authors would like to thank Samuel Ihlenfeldt, Jiayi Deng, and Mohammed Abulela from the Improving Educational Measurement Practice Lab at the University of Minnesota for their helpful comments on an earlier draft.

Author contribution statement: The first author conceived of the presented idea, identified the datasets, conducted descriptive analyses, and drafted the majority of the article. The second author ran the hierarchical model analyses and assisted with writing the method section. All authors interpreted findings and conducted critical revisions of the article throughout the review process. Final approval of the version to be published was made by all authors.

Correspondence concerning this article should be sent to Joseph A. Rios, University of Minnesota, 56 E. River Road, 164 Education Sciences Building, Minneapolis, MN 55455. Email: jrios@umn.edu

Cite as: Rios, J.A., \& Soland, J. (2022). An investigation of item, examinee, and country correlates of rapid guessing in PISA. International Journal of Testing. Advanced online publication. https://doi.org/10.1080/15305058.2022.2036161 


\begin{abstract}
The objective of the present study was to investigate item-, examinee-, and country-level correlates of rapid guessing (RG) in the context of the 2018 PISA science assessment. Analyzing data from 267,148 examinees across 71 countries showed that over $50 \%$ of examinees engaged in $\mathrm{RG}$ on an average proportion of one in 10 items. Descriptive differences were noted between countries on the mean number of $\mathrm{RG}$ responses per examinee with discrepancies as large as $500 \%$. Country-level differences in the odds of engaging in RG were associated with mean performance and regional membership. Furthermore, based on a two-level cross-classified hierarchical linear model, both item- and examinee-level correlates were found to moderate the likelihood of RG. Specifically, the inclusion of items with multimedia content was associated with a decrease in RG. A number of demographic and attitudinal examinee-level variables were also significant moderators, including sex, linguistic background, SES, and self-rated reading comprehension, motivation mastery, and fear of failure. The findings from this study imply that select subgroup comparisons within and across nations may be biased by differential test-taking effort. To mitigate RG in international assessments, future test developers may look to leverage technology-enhanced items.
\end{abstract}

Keywords: rapid guessing, test-taking, effort, international assessments 


\section{An Investigation of Item, Examinee, and Country Correlates of Rapid Guessing in PISA}

As many countries attempt to develop or sustain knowledge-based economies in which technical specialization is of increased importance, the production of human capital has grown as a priority internationally. To promote human capital production, countries have sought to identify and promote effective practices that foster the necessary competencies required by the knowledge society (OECD, 2017). This has been partly accomplished by engaging in international education studies in which country-level comparisons of performance on assessments of critical competencies allow governments to evaluate their students' competitiveness globally.

Although these assessments have the potential to inform educational stakeholders with national-level information concerning areas of student strengths and weaknesses as well as correlates of student success, it is assumed that performance is reflective of the assessed competencies. However, given the complexities in sampling, test development, test administration, and scoring, there have been growing concerns about the validity of inferences made from these assessments (e.g., Hambleton et al., 2004; Matsumoto \& van de Vijver, 2011; Pizmony-Levy et al., 2014). One validity issue that has received increased attention in the literature is whether country-level differences in performance can be attributed to differential test-taking effort (e.g., Gneezy et al., 2017). That is, can we assume that examinees from all participating countries use comparable effort when taking these assessments given potential differences in attitudes around, emotions towards, and behaviors on tests (see Harris \& Brown, 2016)? If this assumption is untenable, observed performances may not be reflective of true differences, and thus, may lead to inaccurate comparative inferences (Rios, 2021a).

To assist in mitigating these invalid inferences, the focus of this study is on investigating correlates of test-taking effort on an international science assessment administered as part of the 
Programme for International Student Assessment (PISA). The goal of this research is to not only document the potential presence of differential test-taking effort as a source of constructirrelevant variance for international comparisons, but also to provide recommendations to test developers and users about item, examinee, and country-level characteristics that may be associated with decreased test-taking effort. Specifically, we model item-level measures of effort as a function of related item, student, and country covariates. In the sections that follow, we provide a conceptual definition of test-taking effort, review prior literature that has examined correlates of this construct in both domestic and international assessment contexts, and present a rationale for the current study.

\section{Test-Taking Effort}

The Expectancy-Value Theory of achievement motivation is the framework that is most often used to explain test-taking effort. Specifically, this theory proposes that effort is moderated by two factors, perceived task value and expectancy of success on the task (Pintrich \& Schunk, 2002). The former can be further broken down into four components: attainment value (importance), intrinsic value (enjoyment), utility value (usefulness of the task), and cost (emotional cost or effort). These combined components are assumed to impact effort based on an individual weighing the personal benefits and costs associated with engaging in a task. Thus, when the costs of partaking in the task outweigh the benefits, individuals may disengage. This is supported by prior research, which has shown that individual effort tends to decrease more often for assessment activities that are perceived to provide little to no personal consequences for examinees (low-stakes testing contexts; e.g., state accountability tests) when compared to those that are thought to offer personal benefits (e.g., Wise \& DeMars, 2005). 
Although task value has been found to be the strongest predictor of effort in testing activities (Penk \& Schipolowski, 2014), prior research has demonstrated that effort can fluctuate across a testing experience (e.g., Wise \& Kingsbury, 2016), suggesting that engagement may be dependent on more than perceived value. As such, researchers have hypothesized that perceived probability of success is also an important moderator of effort (Penk \& Schipolowski, 2014). Specifically, some examinees may decrease their effort when encountering an item or set of items in which the cost of expending effort may be too great given the perceived probability of success. This may be based on the recognition that the requisite knowledge, skills, or abilities to be successful are not possessed (Wise, 2017).

Regardless of the reason for decreased test-taking effort, it has been shown to produce scores that generally underestimate ability by an average of 0.59 standard deviations (Wise \& DeMars, 2005). This occurs as examinees may provide noneffortful responses (i.e., providing an answer with intentional disregard for the item content) that are unreflective of the assessed knowledge, skills, and/or abilities. One form of noneffortful responding that has received considerable attention in the literature for multiple-choice cognitive assessments is rapid guessing (RG). ${ }^{1} \mathrm{RG}$ arises when examinees respond in so little time that they would not be able to fully read the item stem and response options, process the information presented, solve the problem, and provide an answer. Such behavior is identified using item response times and has been shown to be both associated with self-reported effort and equivalent to random responding (Wise, 2017). A failure to consider the presence of RG can bias: (a) treatment effects (e.g., Osborne \& Blanchard, 2011); (b) achievement gains (e.g., Wise \& DeMars, 2010); (c) value-

\footnotetext{
${ }^{1}$ Although there are multiple approaches that have been utilized to operationalize potential low test-taking effort, such as self-reported diligence, multivariate outlier analysis, and response pattern inconsistencies (see Meade \& Craig, 2012), this study utilized rapid guessing as it is the most used proxy employed for multiple-choice cognitive assessments, which is the focus of this study (see Silm et al., 2020).
} 
added estimates of teacher effectiveness (e.g., Jensen at al., 2018); and (d) achievement gap estimates (e.g., Soland, 2018a). In an attempt to mitigate incorrect inferences made from lowstakes testing contexts, researchers have looked to provide test development and score interpretation recommendations by studying individual and assessment contexts that are associated with low effort.

\section{Correlates of Test-Taking Effort}

\section{Domestic Assessment Contexts}

Across a number of domestic assessment contexts (i.e., tests administered to populations in a single country), test-taking effort has been found to be moderated by both item/test and examinee characteristics. In regard to the former, examinees have been shown to disengage at higher rates based on: (a) testing mode (e.g., Kroehne et al., 2020); (b) the absence of a proctor during test administration (e.g., Kroehne et al., 2020; Rios \& Liu, 2017); (c) item length (e.g., Wise, 2006; Wise et al. , 2009); (d) item difficulty (e.g., Pintrich \& Schunk, 2002); (e) item position (e.g., Wise, 2006; Wise et al., 2009; Wolf et al., 1995); and (f) item response option type (e.g., DeMars, 2000). Furthermore, meaningful differences in effort have been documented between sex (females generally display greater effort; see DeMars et al., 2013), age (effort is generally greater for younger; e.g., DeMars, 2007), ethnicity (Black students disengage more than their White counterparts; e.g., Soland, 2018b), linguistic proficiency (linguistic minorities tend to engage less with the assessment; e.g., Setzer et al., 2013), personality trait (effort has been found to differ based on individuals' level of conscientiousness; e.g., Freund \& Holling, 2011), academic ability (lower ability students disengage more than their higher ability counterparts; e.g., Penk et al., 2014; Rios et al., 2017), and academic engagement/socialemotional outcomes (Soland et al., 2019). 
These findings have prompted a number of recommendations in the literature to engender effort and inhibit inattention. First, researchers have suggested that testing programs can increase examinee effort by administering tests via computers. This can be accomplished by allowing for the opportunity to target the difficulty of an item (using adaptive testing) to examinees' ability as well as to include interactive item types into the assessment (e.g., Wise, 2019). Tailoring item difficulty and including non-static items on an assessment may respectively increase perceived probability of success (i.e., examinees may not receive items that are too difficult) and enjoyment (i.e., interactive items may be of greater enjoyment to examinees; e.g., Wise et al., 2021).

In addition, evidence suggests benefits to testing programs employing human proctors and/or automated warning messages to encourage effort in mitigating low test-taking effort (e.g., Lau et al., 2009; Wise et al., 2019). This finding is based on prior research demonstrating that when students know they are being monitored either by another human or remotely via computer software, their effort tends to increase (e.g., Wise et al., 2006). Regardless of the test mode and administration procedures employed, it has been proposed that test developers should avoid lengthy item stems, minimize the number of response options, and limit the number of openended items included on low-stakes assessments to decrease the perception that items are too mentally taxing (Wise, 2006; Wise et al., 2009).

\section{International Assessment Contexts}

Although extensive research has been conducted to investigate correlates of test-taking effort in single-country contexts, there has been far less research for international assessments; however, these limited studies have provided interesting insights. For one, numerous studies have illustrated the presence of differential test-taking effort across countries (e.g., Boe et al., 
2002; Debeer et al., 2014; Goldhammer et al., 2016; Zamarro et al., 2018). As an example, Zamarro et al. (2018) found that on the 2009 administration of the PISA, upwards of $38 \%$ of variation in country-level scores could be explained by differential effort across countries and not simply by differences in knowledge.

Second, although differential test-taking effort has been illustrated by a number of authors, less research has looked to explain why these differences exist, particularly for examinee-level characteristics. The limited research on this topic has found that ability may be associated with test-taking effort, with higher rates of disengagement occurring for lower performing examinees and countries (Debeer et al., 2014; Rios \& Guo, 2020). In addition, both sex (females were more engaged) and age (younger students were more engaged) have been shown to be significantly associated with effort; however, Rios and Guo (2020) found that these associations may be non-invariant across countries in certain assessment contexts.

Third, research examining item- and test-level correlates of test-taking effort has similarly been limited. Zamarro et al. (2018) found an association between item position and effort, with effort tending to dissipate as the test progressed. In terms of test-level correlates, both perceived test difficulty and content domain has been found to be significantly associated with effort. As an example, Rios and Guo (2020) showed that, in some countries, examinees who rated an assessment to be "very difficult" were found to disengage on significantly more items than examinees who rated the same assessment to be of "average" or "no" difficulty. Furthermore, Goldhammer et al. (2016) found that when comparing effort across countries, higher rates of disengagement were generally observed on a problem-solving domain when compared to literacy and numeracy domains, suggesting that content area may be an important correlate. 


\section{Study Rationale}

Our literature review suggests that, though there has been extensive research investigating correlates of test-taking effort in domestic testing contexts, far less research has examined international assessments. As a consequence, it is unclear whether correlates found in single-country contexts generalize to disparate international populations. Furthermore, many of the investigations that have examined correlates of effort in international assessment contexts have failed to consider the multi-level nature of data by focusing on either item or examinee correlates (e.g., Goldhammer et al., 2016; Rios \& Guo, 2020). In addition, outside of the work conducted by Debeer et al. (2014), there have been limited attempts to understand how country level characteristics may impact test-taking effort. Thus, a failure of prior studies to control for correlates at multiple levels may limit our understanding of the factors associated with low testtaking effort on international assessments.

To address these limitations, the objective of this study was two-fold. First, we examined whether differential RG was present across countries participating in the 2018 science domain administration of the PISA. Second, we then modeled RG responses observed on the assessment as a function of item-, examinee-, and country-level characteristics using multiple hierarchical linear models. These objectives were addressed via the following research questions:

1. Are there country-level differences in the average proportion of RG responses by examinee?

2. If so, how much of the variance in RG is at the examinee- versus country-level?

3. What examinee- and country-level factors are most predictive of RG on a given item?

4. Are the same examinee-level factors predictive when also accounting for item characteristics? Which item-level characteristics are predictive of RG? 
Findings from this study have the potential to inform future test development of international science assessments by considering item-level characteristics that are associated with RG. Furthermore, observed examinee- and country-level subgroup differences in RG may suggest to both measurement specialists and score users the importance of considering the potential presence of differential effort prior to making score comparisons.

\section{Method}

\section{Measure}

PISA is an international assessment measuring 15-year-old's knowledge and skills in reading, mathematics, and science. It is administered to member and non-member countries participating in the Organization for Economic Co-operation and Development (OECD) every three years. In the 2018 administration, each student was administered a cognitive assessment that included at minimum two of four primary domains in reading, math, science, and global competence. However, the focus of this study was on the science literacy (i.e., knowledge of science and of science-based technology) domain, which is comprised of three competencies: explaining phenomena scientifically, evaluating and designing scientific enquiry, and interpreting data and evidence scientifically. The rationale for examining the science domain is two-fold. First, most research to date in the RG literature has focused on non-science content areas (e.g., Abdelfattah, 2007; Rios et al., 2014; Smith et al., 2013; Wise, 2006; Wise, 2015; Wise et al., 2020). Second, given that the objective of this analysis was to investigate RG as a proxy of low test-taking effort, it was necessary to choose a domain with a large number of multiple-choice items (RG has primarily been operationalized in the context of such items; see Wise, 2017). Of the four domains administered on the 2018 PISA, the science domain provided the largest sample of such items. 
In the 2018 administration, science literacy was administered to $41 \%$ of all participants taking the computer-based version of PISA. A matrix sampling design (i.e., each examinee is randomly administered a subset of items) was employed in which students received at least one of six science item clusters (33\% of students were administered two science item clusters). Each cluster was comprised of between 18 to 20 items, and was designed to take 30 -minutes to administer. As we utilized response latencies as a proxy of $\mathrm{RG}$, the focus of this analysis was on the computer-based administration of the science domain. In addition to the science domain, individuals answered questionnaires that covered a variety of constructs including family, schooling, and non-cognitive/metacognitive constructs (e.g., motivation and self-efficacy scales). Each student was tested for approximately two hours, including both cognitive and questionnaire items.

\section{Sample}

Data were collected for 606,627 students in 79 countries aged between 15 years and 3 months and 16 years and 2 months, with country-level sample sizes ranging from 2,016 to 22,653 . In each participating country, the desired sample size was a minimum of 5,000 nationally representative students from at least 150 schools; however, in some small countries (e.g., Iceland), such a target may have been infeasible. Students were excluded if possessing: (a) a moderate to severe permanent physical, cognitive, behavioral, or emotional disability that would not allow them to participate in testing; (b) and/or limited proficiency in the assessment language. The final sample consisted of 267,148 examinees from 71 countries that were administered at least one science item. The 71 countries represented were primarily distributed across Europe $(n=39)$ followed by Asia/Oceania $(n=13)$, the Americas $(n=11)$, and Africa/Middle East $(n=9)$. 


\section{Variables}

Below we discuss the correlates of RG investigated separately by item-, examinee-, and country-level.

\section{Item-level Correlates}

Five item-level characteristics were investigated as correlates of RG: (a) stimulus type; (b) item type; (c) item position within cluster; (d) item difficulty; and (e) cognitive demand.

Stimulus Type. Nearly all items on the science assessment were part of testlets that shared a common stimulus material. The stimulus could consist of a brief written passage or text accompanying a table, chart, graph or diagram. However, of interest to this study was whether the stimulus material was presented in a static or non-static fashion, with the latter consisting of animations or interactive simulations. Of the 115 PISA science items, approximately $22 \%$ were interactive. It was hypothesized that non-static items would be associated with lower levels of RG, as these items may elicit greater appeal to examinees (Wise, 2019).

Item Type. Three item types were included in each cluster: single-select multiple-choice (SMC), complex multiple-choice (CMC), and open-response items. SMC items required students to choose one of four response options as the correct answer or provide an answer that was a selectable element within a graphic or text. CMC items necessitated that students select more than one correct answer out of a set of response options that numbered greater than four. These item response options could be presented as a series of related "yes/no" questions, the selection of more than one response from a list, the completion of a sentence using multiple drop-down choices, or "drag-and-drop" responses. For students to receive credit on the latter set of items, all correct responses had to be provided. Both SMC and CMC items were computer scored. Finally, 
in the open-response items, students were given the opportunity to answer questions via short text responses, which were human-graded based on a scoring rubric or computer-scored.

Across the 115 science items administered, 33 were SMC, 47 were CMC, and 35 were open-ended items. For the purposes of this study, RG rates were compared across each of these item types. Given the findings of prior literature, it was hypothesized that higher rates of RG would be observed for CMC and open-response items, due to the increased cognitive demands required of such item types (e.g., DeMars, 2000).

Item Position Within Cluster. To investigate whether test-taking effort dissipated based on item position as previous research has shown (Wise et al., 2009), each item's sequence number in their respective item cluster was included as an independent variable. As each item cluster consisted of between 18 to 20 items, item positions ranged from a minimum of one to a maximum of 20. However, given that a spiral design was employed in the PISA administration, item cluster positions across examinees could differ.

Item Difficulty. To account for the potential association between item difficulty and RG, each item's difficulty was included as an independent variable. In this study, item difficulty was measured based on the proportion of examinees across the international sample that correctly answered the item of interest (i.e., proportion correct rates were calculated for the entire worldwide sample). For the 115 science items administered, item difficulty was found to range from .10 to .85 , with a median of $.43(\mathrm{SD}=.17)$.

Cognitive Demand. All items targeted one of three cognitive demand levels based on an adapted version of Webb's Depth of Knowledge Grid (Webb, 1997): low, medium, or high. Low cognitive demand items required the recollection of one piece of information (e.g., recalling a fact, term, principle or concept or locating a single point of information from a graph or table). 
Medium demand items assessed the ability to interpret or use simple data sets or graphs, organize or display data, and use and apply conceptual knowledge to describe or explain phenomena. Items requiring the recollection of more than one piece of knowledge based on a comparison or an evaluation of their competing relevance were of high cognitive demand. Across the 115 items examined, approximately $27 \%, 64 \%$, and $9 \%$ were of low, medium, and high cognitive demand, respectively. It was hypothesized that $\mathrm{RG}$ would increase as the cognitive demand of items increased (Wise, 2017).

\section{Examinee-level Correlates}

Eight examinee-level characteristics were investigated as correlates of RG: (a) biological sex (hereon referred to as sex); (b) language spoken at home; (c) immigrant background; (d) index of economic, social, and cultural status (hereon referred to as socioeconomic status [SES]); (e) self-rated reading competency; (f) self-efficacy; (g) motivation to master tasks; and (h) fear of failure. These variables were based on either simple or scaled indices. The former refers to variables constructed through the arithmetic transformation or recoding of one or more items, while the latter consisted of variables constructed through the scaling of multiple items using a two-parameter item-response model or generalized partial credit model.

Sex. Numerous studies have shown sex differences in test-taking effort on low-stakes tests, with females showing greater engagement (e.g., DeMars et al., 2013). To investigate if this association was significant in the assessment context examined, student self-reported sex was included as a dichotomous variable (male $=0$ and female $=1$ ).

Language Spoken at Home. As part of the student questionnaire, students were asked what language they usually speak at home. From this question a dichotomous variable was created with the following levels: (a) language spoken at home is the same as the language of 
assessment; and (b) home language differs from the assessment language. As noted prior, previous research has shown significant differences in test-taking effort based on whether examinees were assessed in their native language (e.g., Setzer et al., 2013).

Immigrant Background. For students and their parents (both mother and father), questionnaire data was collected on their country of birth. These variables were combined to designate three levels of student immigrant background. Specifically, students were labeled as a native if they and at least one parent were born in the country where the student was tested. For those students with both parents born outside of the country, there were two designations: firstand second-generation immigrant. The former was designated for students born outside the country of assessment, while the latter was the classification for students born in the country.

SES. A scaled index of economic, social, and cultural status was derived based on parents' highest level of education, parents' highest occupation status, and home possessions, with the latter serving as a proxy of family wealth. For greater details, the reader is referred to $\operatorname{OECD}(2019 a)$

Perceived Reading Competency. Students were asked to answer the following three statements using a four response category scale ("not at all", "very little", "to some extent", and "a lot") to create a scaled index variable of perceived competence in reading: "I am a good reader"; "I am able to understand difficult texts"; and "I read fluently". This variable was included as a moderator given that prior research has shown a significant association between reading competency and $\mathrm{RG}$ (Debeer et al., 2014).

Self-Efficacy. A general self-efficacy composite scaled variable was derived based on the extent of agreement ("strongly disagree", "disagree", "agree", and "strongly agree”) to the following statements: "I usually manage one way or another"; "I feel proud that I have 
accomplished things"; "I feel that I can handle many things at a time"; "My belief in myself gets me through hard times"; and "When I'm in a difficult situation, I can usually find my way out of it”. Soland et al. (2019) found the self-reported self-efficacy is strongly associated with RG in a U.S. context.

Motivation to Master Tasks. A scaled index variable was created to ascertain students' motivation to master tasks based on the extent to which they agreed or disagreed ("strongly disagree", "disagree", “agree”, “strongly agree”) on the following four statements: "I find satisfaction in working as hard as I can"; "Once I start a task, I persist until it is finished"; "Part of the enjoyment I get from doing things is when I improve on my past performance"; and "If I am not good at something, I would rather keep struggling to master it than move on to something I may be good at". In U.S. samples, achievement motivation has been shown to be associated with RG (Soland et al., 2019).

Fear of Failure. Three four-point (“strongly disagree”, “disagree”, "agree”, "strongly agree") Likert items were administered to students to create a fear of failure scaled index variable. These three questions included: "When I am failing, I am afraid that I might not have enough talent"; "When I am failing, this makes me doubt my plans for the future"; "When I am failing, I am afraid that I might not have enough talent". Similar to the variable above, this moderator was included because it is strongly related to achievement motivation, which is associated with RG (Soland et al., 2019).

\section{Country-level Correlates}

Four country-level variables were included: (a) mean science performance; (b) sex differences in science performance; (c) OECD membership; and (d) region. 
Mean Science Performance. As Debeer et al. (2014) demonstrated higher rates of test disengagement for lower performing countries, mean science performance was included as an independent variable, which was entered as the mean scale score for each country. Following the findings from prior literature, it was hypothesized that mean science performance and RG would be negatively associated.

Sex Differences in Science Performance. To examine if country-level sex gaps in science performance could be attributed to test-taking engagement, the mean scale score difference between females and males (a positive score reflected higher performance for females) was entered as an independent variable. This information was obtained from OECD (2019b).

OECD Membership. A dichotomous variable for 2018 OECD membership was included as a proxy for a country's economic development. This allowed us to examine variability in test-taking engagement by differences in economy. Economies that were key OECD partners (e.g., Brazil, China, Indonesia) were coded as an OECD member, which resulted in 39 countries belonging to this category.

Region. Little is known about potential regional differences in test-taking effort. Such a concern may arise due to regional differences in practices and cultures around testing. To examine this issue, a country's region was coded based on OECD regional initiatives, which included the following regions: the Americas (North, Central, and South America along with the Caribbean), Europe, Asia and Oceania, and Middle East and North Africa.

\section{Outcome Variable}

Prior to investigating correlates of RG, it was first necessary to calculate our dependent variable, which was the occurrence of RG at the examinee-by-item level. This was done by 
evaluating item response times based on examinee's last item visit (calculated as the difference between timestamps of the last item loading event and an item ending) and classifying RG based on the normative threshold (NT10) procedure. $^{2}$ This method was first proposed by Wise and Ma (2012), and it classifies a rapid response as any response provided in less than $10 \%$ of the mean item response time for the sample of interest. ${ }^{3}$ This was done separately for each country, given differences in language of test administration, similar to the work conducted by Rios and Guo (2020). The NT10 procedure has been employed across multiple studies, and has been shown to demonstrate adequate validity evidence in applied analyses (for further details, see Wise \& Ma, 2012). A dichotomous variable (present/not present) for RG was created for every examinee by item interaction.

\section{Multilevel Analyses}

Research Question 1. Information on RG behavior was examined by calculating the average proportion of RG responses per examinee by country. These proportions were compared to examine descriptive differences across countries and regions.

Research Question 2. To examine the partitioning of variance in RG across item, examinee, and country levels, we began by fitting a three-level random-intercepts model. This was done by nesting rapid guesses for item $i$ within examinee $j$ nested within country $k$ for $\mathrm{y}_{i j k}$, which is a binary variable, with $1=\mathrm{RG}$ and $0=$ an effortful response. Given the binary nature of

\footnotetext{
${ }^{2}$ In 2020, PISA uploaded an additional file, cognitive items total time/visits data file, which includes a testing time variable based on the total number of item visits (i.e., examinees were allowed to visit an item multiple times). However, analyses demonstrated that in $93 \%$ of cases ( $n=47,278,588$ item visits), examinees visited items only once. Further analyses demonstrated a nearly identical median response time per item distribution when calculated based on examinee's last visit and their total visits for a given item (see https://www.oecd.org/pisa/data/pisa2018technicalreport/PISA2018-TechReport-Annex-K.pdf). Thus, our utilization of response times based on the last item visit likely had minimal influence on the presented results.

${ }^{3}$ Although Wise and Ma (2012) proposed establishing an upper-bound threshold of 10 seconds when the mean item response exceeded 10 seconds, no such threshold was utilized in this study. Rapid responses were simply defined as $10 \%$ of the mean item response time for each individual country.
} 
the data, we used a probit link, and thus modeled $\mathrm{y}_{i j k}^{*}$, or the continuous normal variable assumed to underlie the observed coarsened variable. Given the probit approach, the level-1 residual is arbitrarily scaled to have a variance of one. The specific model consisted of:

$$
\mathrm{y}_{i j k}^{*}=\pi_{0 j k}+e_{i j k} .
$$

The level-2 model for item $i$ within examinee $j$ then becomes

$$
\pi_{0 j k}=\beta_{00 k}+r_{0 j k}
$$

while the level-3 model for country $k$ is

$$
\beta_{00 k}=\gamma_{000}+u_{00 k}
$$

The variance component was specified as:

$$
e_{i j k} \sim N\left(0, \sigma^{2}\right), \quad r_{0 j k} \sim N\left(0, \tau_{00}^{(2)}\right), \quad u_{00 k} \sim N\left(0, \tau_{00}^{(3)}\right),
$$

where $\sigma^{2}$ is constrained to equal 1 . We then produced several decompositions of the variance, including the proportion of variance at the item-,

$$
\frac{\sigma^{2}}{\sigma^{2}+\tau_{00}^{(2)}+\tau_{00}^{(3)}}
$$

examinee-,

$$
\frac{\tau_{00}^{(2)}}{\sigma^{2}+\tau_{00}^{(2)}+\tau_{00}^{(3)}}
$$

and country-level:

$$
\frac{\tau_{00}^{(3)}}{\sigma^{2}+\tau_{00}^{(2)}+\tau_{00}^{(3)}}
$$

In addition, we decomposed the between- versus within-country variance (intraclass correlation coefficient or ICC) using the following equation:

$$
\frac{\tau_{00}^{(3)}}{\tau_{00}^{(2)}+\tau_{00}^{(3)}}
$$


which is interpretable as the correlation between the mean rapid guess for any two students in the same country.

Research Question 3. When looking at student- and country-level predictors, we built on the model presented above in Equations 1-3. This specific model consisted of:

$$
\mathrm{y}_{i j k}^{*}=\pi_{0 j k}+e_{i j k}
$$

The level-2 model for item $i$ within examinee $j$ then becomes

$$
\begin{array}{r}
\pi_{0 j k}=\beta_{00 k}+\beta_{01 k} \text { ESCS }_{j k}+\beta_{02 k} \text { immigrant }_{j k}+\beta_{03 k} \text { female }_{j k}+\beta_{04 k} \text { reading }_{j k} \\
\beta_{05 k} \text { fearfail }_{j k}+\beta_{06 k} \text { efficacy }_{j k}+\beta_{07 k} \text { motivation }_{j k}+\beta_{08 k} \text { language }_{j k}+r_{0 j k},
\end{array}
$$

where $E S C S_{j k}$ is a continuous variable representing an examinee's SES; immigrant $_{j k}$ is a dummy-coded variable representing whether an examine was born outside the country where they were tested (examinees born within the country were the reference); female $_{j k}$ is a dummycoded variable representing whether an examine was female (males were the reference); reading $_{j k}$ is a continuous variable representing an examinee's self-rated reading competency; fearfail $_{j k}$ is a continuous variable representing an examinee's self-rated fear of failure; efficacy ${ }_{j k}$ is a continuous variable representing an examinee's self-reported self-efficacy; motivation $_{j k}$ is a continuous variable representing an examinee's self-reported motivation to master tasks; and language ${ }_{j k}$ is a dummy-coded variable representing whether an examinee was tested in their language spoken at home (native language examinees were the reference).

Finally, the level-3 model for country $k$ is:

$$
\begin{gathered}
\beta_{00 k}=\gamma_{000}+\gamma_{001} \text { meanscor }_{k}+\gamma_{002} \text { gendergap }_{k}+\gamma_{003} \text { OEC }_{k}+ \\
\gamma_{004} \text { Americas }_{k}+\gamma_{005} \text { Asia }_{k}+\gamma_{006} \text { Africa }_{k}+u_{00 k} \\
\beta_{01 k}=\gamma_{010}+u_{01 k} \\
\beta_{02 k}=\gamma_{020}+u_{01 k}
\end{gathered}
$$




$$
\begin{gathered}
\beta_{03 k}=\gamma_{030}+u_{03 k} \\
\beta_{04 k}=\gamma_{040}+u_{04 k} \\
\beta_{05 k}=\gamma_{050}+u_{05 k} \\
\beta_{06 k}=\gamma_{060}+u_{06 k} \\
\beta_{07 k}=\gamma_{070}+u_{07 k} \\
\beta_{08 k}=\gamma_{080}+u_{08 k},
\end{gathered}
$$

where meanscore $e_{k}$ is a variable capturing the mean scaled science score for each country; gendergap ${ }_{k}$ is the mean scaled science score difference by sex for each country; $O E C D_{k}$ is a dummy-coded variable representing OECD country membership (no country membership served as the reference group); Americas $s_{k}$ is a dummy-coded variable representing a country's regional membership in the Americas (Europe served as the reference group); Asia $_{k}$ is a dummy-coded variable representing a country's regional membership in the Asia or Oceania (Europe served as the reference group); frica $_{k}$ is a dummy-coded variable representing a country's regional membership in Africa or the Middle East (Europe served as the reference group). Given that the model treats all of the examinee-level effects as random, it provides a country-level mean for those examinee-level covariates, as well as estimates of their within-country variances. Finally, the variance component specification for the model is:

$$
e_{i j k} \sim N\left(0, \sigma^{2}\right), \quad r_{0 j k} \sim N\left(0, \tau_{00}^{(2)}\right), \quad \boldsymbol{u}_{00 k} \sim M V N\left(\mathbf{0}, \boldsymbol{\tau}_{00}^{(3)}\right) .
$$

Research Question 4. A different model specification was employed to examine how the inclusion of item characteristics in the model affects examinee-level predictors. We made this choice because rapid guesses are more accurately thought of as varying at the examinee-by-item level, whereas the item characteristics only varied at the item level. That is, the model assumes that rapid guesses are cross-classified within items and examinees, where an item might be, say, the first question on the science test. Such a model has been used previously to model item responses in an IRT framework for the PISA data (Fox \& Glas, 2016). 
Under this cross-classified structure, at level-2, there are separate predictors for items, $c$, and people, $j$. The new item-level predictors include: instsim $_{c}$, a dummy-coded variable equal to the inclusion of interactive item stimulus (items with static item stimuli were the reference); seqclust $_{c}$, a continuous variable equal to the sequence item number within its item cluster; open $_{c}$ represents a dummy-coded variable equal to the presence of an open-ended item (reference group is simple multiple-choice items); complex $x_{c}$ represents a dummy coded variable equal to the presence of a complex multiple-choice item (reference group is simple multiplechoice items); $\operatorname{cogmed}_{c}$ is a dummy-coded variable equal to the presence of a medium cognitive demand item (low cognitive demand items were the reference group); $\operatorname{coghigh}_{c}$ is a dummycoded variable equal to the presence of a high cognitive demand item (low cognitive demand items were the reference group); and intcor $_{c}$ is a continuous variable representing item difficulty. There are also separate residuals for examinee $r_{0 j}$ and item $r_{0 c}$.

Beyond this new formulation, one other aspect of the model bears mention. Given this is a probit model, a threshold for whether the item response was a rapid guess is estimated. One might reasonably expect, however, that the threshold should differ by item $c$. Therefore, we used two different specifications, one where we treated the threshold as fixed, the other where we treated it as random and allowed it to vary across items, an approach taken by Fox and Glas (2016).

\section{Results}

\section{Are There Country Differences in RG?}

Across the sample, less than half of examinees (44\%) employed full solution behavior for all items administered. For those examinees that displayed some RG, the average proportion of $\mathrm{RG}$ responses was $.11(\mathrm{SD}=.06)$, while across all examinees this proportion was equal to .06 
$(\mathrm{SD}=.11)$. Figure 1 provides a bar plot of the average proportion of $\mathrm{RG}$ responses per examinee by country. As shown in this figure, large variations were observed across countries, with the highest and lowest proportions respectively represented by the Dominican Republic (.16) and Mexico (.03). Across regions, countries belonging to the Africa/Middle East region possessed the highest average proportion per examinee with $.08(\mathrm{SD}=.03)$ of items receiving an $\mathrm{RG}$ response.

\section{How Much of the Variance in RG is at the Examinee- Versus Country-Level?}

Employing an intercept-only three-level model in which item-level RG responses were nested within students and students were nested within countries showed that the variance in RG was largely explained by within-country differences. Per the ICC in Equation 8, only 9\% of RG response variance was attributed to variability between countries, while the remaining $91 \%$ of variance was ascribed to within country differences. Meanwhile, based on Equations 4-6, nearly two-thirds of the overall variance in $\mathrm{RG}$ was at the item level $(\sim 63 \%)$, followed by examinees $(\sim 34 \%)$, and then countries $(\sim 3 \%)$.

\section{What Examinee- and Country-level Factors are Most Predictive of Whether a Student Rapidly Guesses on a Given Item?}

We next turn to examinee-level characteristics that were found to have a significant association with RG. As shown in Table 1, of the eight independent variables investigated, both demographic and self-reported academic and socioemotional factors were found to be statistically significant. In terms of student demographics, SES, sex, and home language were important predictors, while immigrant status was not. The strongest demographic predictors of RG were home language and sex. Specifically, examinees that did not speak the same language of the administered test at home on average possessed a z-score difference on the outcome that 
was 0.12 units higher than their same language counterparts (suggesting a positive association with RG). Subgroup differences were also observed by sex, with the average occurrence of RG 0.11 SD units higher for males. In addition, examinees with higher SES tended to show less RG, with a one-unit increase in SES associated with a decrease in RG by 0.06 units.

In terms of attitudinal factors, a one-unit increase in self-reported reading comprehension and motivation mastery were both associated with a decrease in RG by 0.06 SD units. Other factors, such as general academic self-efficacy and fear of failure, were not found to be significantly associated with RG (holding all other covariates constant). Given the three-level structure of the model, all coefficients represent country-level means. Notably, the effects of all the student-level demographic and attitudinal factors varied significantly within-country, suggesting that there is likely meaningful variability in their impact on RG at the examinee-level.

Focusing next on country-level predictors, OECD membership and sex gaps in science achievement were not found to be significant moderators; however, statistical differences were noted by country mean performance and regionality. As an example, a one-unit increase in a country's mean performance was associated with a small decrease in RG (.002 SD units), suggesting that higher performing countries on average engaged in RG at a lesser rate (though the practical significance of this result is open to interpretation). Large differences in RG were noted by country region. In particular, when compared to Europe, member states of the Americas showed no significant differences, while the occurrence of RG was significantly higher on average for countries in Asia/Oceania and Africa/Middle East by 0.17 and 0.26 units, respectively.

\section{Are the Same Examinee-level Factors Predictive when also Accounting for Item}

Characteristics? Which Item-level Characteristics are Predictive of RG? 
As shown in Table 2, when examining a two-level cross-classified model, we found that all of the same examinee-level factors were predictive of RG occurrence once accounting for item-level characteristics. Further, when not accounting for country-level factors/clustering, immigrant status and fear of failure were also significant. Concerning the former predictor, immigrant examinees on average possessed a z-score that was $0.04 \mathrm{SD}$ units higher than their non-immigrant counterparts. One possible explanation for this result is that immigrant status is associated with the examinee's country, and is in some way serving as a proxy for country in the model. Similar to the three-level model, the strongest moderators of RG were sex, home language, SES, and reading comprehension, while, academic self-efficacy was not a significant factor. Turning to item-level predictors, all were non-significant, except for one, once accounting for examinee-level factors. Specifically, the inclusion of an interactive stimulus item was associated with a decrease in RG occurrence by $0.24 S D$ units when compared to a noninteractive stimulus.

\section{Discussion}

The objective of the presented study was to examine item-, examinee-, and country-level correlates of RG. In the context of the PISA science assessment, results suggest that RG is a prevalent concern, given that over $50 \%$ of examinees engaged in $\mathrm{RG}$ on upwards of one in ten items; this is a RG rate that is often sufficient to raise concerns about the validity of the observed scores (e.g., Soland, 2018b; Wise, 2015). However, the rate of RG was found to descriptively vary by country, with the mean proportion of RG responses per examinee differing by more than $500 \%$ between some nations.

Model results suggested that these differences were related to two factors. First, examinees from countries with lower mean performance were found to engage in RG at a higher 
rate than those from better performing countries, supporting prior research (e.g., Debeer et al., 2014; Rios \& Guo, 2020). Second, after controlling for country mean performance, regional differences were noted in which the odds of employing RG were higher for examinees in Asia/Oceania and Africa/Middle East compared to examinees in Europe and the Americas. One concern previously raised that may partially explain such findings is related to the equivalence of test translations/adaptations. For instance, prior research has shown that average item length and word frequency usage can differ drastically across languages, even with PISA's implementation of test adaption quality controls (Grisay \& Monseur, 2007). Thus, the differential RG rates observed may be associated with variability in item length (e.g., Wise, 2006; Wise et al. , 2009) and vocabulary comprehension, given that both increase cognitive demand. Future research is needed that investigates this hypothesis.

Further, these results point to the role of sociocultural factors in the assessment process. Specifically, PISA as an assessment system is a cultural product that prioritizes math, reading, and science as universal competencies. However, such an assumption of the importance of these competencies may not be fully accepted by individuals within countries, let alone across all participating nations. In addition, there may also be variability in testing cultures, given that some countries participating in PISA have utilized assessments within their education system more than others have. As such, students, educational professionals, the general public, and policy makers across countries may view assessment results as possessing differential utility in informing educational decision-making (see Eivers, 2010). As noted by Ercikan and SolanoFlores (2016), this may be one reason why examinees across cultures place differential value on performance in international studies, such as PISA. Future research is needed to elucidate how cultural differences in test-taking perceptions and experiences may be associated with examinee 
motivation (see Wigfield \& Eccles, 2000). Regardless of the potential reasons for differential $\mathrm{RG}$, the findings from this study raise concerns about the validity of comparative performance inferences in international contexts based on the questionable assumption that examinees from different cultures/countries put forth equal test-taking effort on international assessments (e.g., Boe et al., 2002; Borghans \& Schils, 2012; Debeer et al., 2014; Gneezy et al., 2017, Goldhammer et al., 2016; Rios \& Guo, 2020; Zamarro et al., 2019).

At the same time, the majority of variance in RG was within- as opposed to betweencountries. For example, both item- and examinee-level correlates were associated with RG. For instance, after controlling for examinee-level correlates, the presence of multimedia item content was associated with significant decreases in RG (though item difficulty, position, type, and cognitive demand were not found to be significant). This finding supports work conducted by Wise et al. $(2009 ; 2021)$, which found that the presence of graphics can improve solution behavior in low-stakes testing contexts. One potential reason for this result is that, although such items may be more visually complex for examinees, their novelty may elicit greater subjective interest in the item, leading to a greater willingness to allocate mental and time resources to engage in solving the problem (Wigfield \& Eccles, 2000). Such reasoning has led many researchers to call for the increased inclusion of technology-enhanced items (TEIs) and gamebased features in assessment to improve examinee engagement (see Lehman et al., 2019). Beyond the significant association of multimedia items, a number of item characteristics previously noted to account for considerable variation in $\mathrm{RG}$ were found to be non-significant in our analysis. These included cognitive domain, item difficulty, and item position within cluster. One potential reason for the non-significant association of the former predictor is that prior researchers have noted the difficulty that test developers have in developing items that align to 
various cognitive levels (Schneider et al., 2013). One reason for this is that item writers must often hypothesize how contextual item features are related to cognitive complexity in the absence of data. Thus, the cognitive domain labels for the items utilized in our analysis may not have been reflective of the complexity experienced during the assessment process across the heterogeneous population sampled. Concerning the item difficulty predictor, one confounding factor in our analysis was that the proportion correct values utilized were based on the international sample, not on individual country’s data. Such an approach likely masked the heterogeneity in the difficulty of items across countries, given the large degree of variance in observed average ability by country. Lastly, although item position has been found to be significantly associated with RG in prior domestic (e.g., Wise, 2006) and international (e.g., Zamarro et al., 2018) contexts, our study found no such effect. One reason for this is likely due to how we defined item position in our analysis. Specifically, this variable was operationalized based on location within a given item cluster. However, as noted, due to the use of a spiral design in the PISA test administration (i.e., examinees were administered different clusters in varying order), the same item could have been administered at different points of the test across examinees. Thus, although our operationalization captured an item's position within a cluster, it may have not accounted for low test-taking effort due to receiving an item under increased cognitive fatigue towards the end of an assessment. These limitations in operationally defining our variables should be taken into consideration when interpreting the non-significant results from our analysis.

Turning to examinee-level correlates, once controlling for item- and country-level characteristics, both demographic and attitudinal variables were significantly associated with RG. In terms of demographic characteristics, sex, linguistic background, and SES were all found 
to be significant moderators. Specifically, males were found to have a higher probability of engaging in RG than females. This finding has been supported in previous studies examining both domestic and international contexts (e.g., DeMars et al., 2013; Rios \& Guo, 2020; Soland, 2018a). One potential reason for these empirical differences is subgroup variation in personality traits. For instance, DeMars et al. (2013) showed that females tend to be higher in conscientiousness and agreeableness, which are positively associated with test-taking effort, while males are higher in work-avoidance, a construct that is negatively related to effort. Although examined in a domestic assessment context, these results may partially explain the observed differences in RG by sex.

An additional demographic variable that moderated the odds of engaging in RG was linguistic group. In particular, examinees tested in a language that differed from the one spoken at home had a higher probability of employing RG than examinees tested in their home language. This finding supports two prior analyses that examined samples collected in domestic (Major Field Test in Business; Setzer et al., 2013) and international contexts (the Survey of Adult Skills; Goldhammer et al., 2016). One potential reason for this finding is that individuals assessed in a second language may have difficulty accessing the item content due to linguistic barriers. When this is the case, their perceived probability of success may be low, which may lead to disengagement on an item, based on the belief that allocating full effort will not be rewarded by a correct answer (Penk \& Schipolowski, 2015; Wise, 2017). This may also be a potential explanation for the tendency of immigrant and low SES examinees to have a higher probability of engaging in RG, particularly given that these examinees tend to face more barriers (e.g., poverty, violence, segregation) to academic success than their non-immigrant/higher SES counterparts (e.g., Suárez-Orozco et al., 2009). Prior research has shown that many students may 
become academically disengaged when faced with these challenges, which is a construct associated with RG in low-stakes testing contexts (see Soland et al., 2019).

Besides demographic characteristics, a number of individual attitudinal variables were also found to moderate RG. These variables included self-rated reading comprehension, motivation mastery, and fear of failure. With respect to the former variable, a negative association between reading comprehension and likelihood of engaging in RG was observed, which supports previous work conducted by Debeer et al. (2014). One potential explanation for this finding is that examinees may decide to not fully engage and/or solve a problem that requires a skill that they feel they are less than proficient, because the cost of doing so outweighs the expectancy of success (Penk \& Schipolowski, 2015). This is one reason why some researchers have argued that RG is strongly related to examinee ability (e.g., Rios et al., 2017).

Related to persisting when confronted with a challenge, the findings indicated that selfrated motivation mastery was related to RG. Specifically, as would be expected, the likelihood of employing RG was lower for examinees who indicated that they have a tendency to persist when presented with challenging problems. This result speaks to the important interplay between task value and expectancy of success in examinees' achievement motivation (Penk \& Schipolowski, 2015), and the importance of psychological factors in explaining individual differences in RG (e.g., Freund \& Holling, 2011). Notably, self-efficacy was not found to be significant, a result that does not jibe with findings in other studies (e.g., Soland, 2018). This discrepancy could be due to subject differences (science versus math) or to international versus domestic contexts, and should be investigated further.

\section{Limitations and Future Research}

The results from the current study should be interpreted in light of the fact that correlates of RG were only examined for the science PISA assessment - a content area that has been 
understudied in the RG literature. Thus, our findings may not generalize to other content areas or age groups; however, a strength of this analysis is the cross-cultural implications that it may have for the examined assessment context. Furthermore, RG was categorized using only one RT threshold procedure, the NT10 method. Although this is the most popular approach utilized in research settings (see Rios \& Deng, 2021), it has been criticized as being perhaps too conservative in its identification rates (see Kroehne et al., 2020). Thus, it is possible that the number of RG responses may have been lower than if using other methods. With that said, the true rate of $\mathrm{RG}$ in operational settings is unknowable, given the use of response times as a proxy of test-taking behavior (see Rios, 2021b).

Additionally, although various examinee-level characteristics were examined as correlates of RG, examinee academic ability could not be investigated as a potential moderator, given its unavailability in the dataset. This association has implications for how practitioners handle RG responses after they have been identified. To date, two common filtering approaches, response- and examinee-level filtering, either treat RG responses as missing data or listwise delete data for examinees engaging in RG for a pre-defined number of items (see Rios et al., 2017). In both cases, it is assumed that RG is unrelated to examinee ability. When this assumption is untenable, which has been shown to be the case in some applied contexts (e.g., Deribo et al., 2020; Rios et al., 2017; Wise et al., 2009), filtered scores can be biased to a greater extent than when including RG responses in scoring (Rios et al., 2017; Rios \& Soland, 2021). Hence, the findings from this study cannot provide a clear recommendation of the best filtering approach in the PISA context, due to the uncertainty in whether the relationship between ability and RG is consistent across countries. Future research is needed that provides alternative 
filtering/modeling approaches in cases in which response- and/or examinee-level filtering may be inappropriate in handling RG responses (e.g., Deribo et al., 2020).

\section{Implications for Practice}

The findings from this study have two implications for score users. First, given the differences in RG by sex, linguistic, and SES subgroups, it is vital that potential aberrant responding is identified and filtered/modeled prior to making comparative inferences (for recommendations on effective approaches, see Deribo et al., 2020; Rios et al., 2017; Wise, 2017). Although this sentiment is supported by the Standards for Educational and Psychological Testing (American Educational Research Association et al., 2014), most testing programs do not operationally evaluate RG prior to analyzing and interpreting data (Wise \& Kuhfeld, 2021). By not doing so, score users may undermine the utility of international assessments, such as PISA, which provide the opportunity to identify achievement gaps at the national- and/or regional-level in an effort to prescribe ameliorative educational policy reform.

Second, although RG may be pervasive in many low-stakes testing contexts, such as those in international education studies, the item- and examinee-level correlates investigated in this study point to two potential test development efforts that can be made to mitigate this invalid behavior. To begin with, test developers might consider minimizing unnecessary reading load and complexity, since examinees with lower self-rated reading comprehension were found to be more prone to engage in RG, supporting prior research showing an association between word length and RG (Wise, 2006; Wise et al., 2009). Although this recommendation follows best practice guidelines (Haladyna \& Downing, 1989), recent reviews of operational testing programs suggest that current items often include superfluous information that does not improve measurement of the intended construct (Haladyna et al., 2019). Reducing linguistic complexity 
may not only benefit the reduction of RG, but may also advance the validity of score-based inferences for examinees tested in their non-native language (e.g., Noble et al., 2020) - a group found to engage in $\mathrm{RG}$ at higher rates than their native language counterparts.

In addition, test developers may look to include items with interactive stimuli on lowstakes tests, as data from this study suggest that such items reduce RG when compared to those with static content. This supports prior literature that has called for improving test-taking effort by utilizing TEIs to make tests more intrinsically interesting for examinees (Lehman et al., 2019; Wise et al., 2021). In doing so, test developers should work to ensure that TEIs improve construct representation and minimize construct-irrelevant variance associated with usability and accessibility issues that may arise (Russell \& Moncaleano, 2019). This work could be addressed by engaging in cognitive interviews with individuals from the targeted population of interest to investigate their interactions and engagement with newly developed TEIs. If created appropriately, TEIs may also enhance construct representation by improving the authenticity of measurement. Taken together, test developers may mitigate RG by minimizing unnecessary cognitive load and leveraging technology to better measure and engage examinees. These efforts coupled with appropriate identification and filtering/modeling of RG responses can improve score-based inferences from low-stakes testing contexts, such as PISA. 


\section{References}

Abdelfattah, F. A. (2007). Response latency effects on classical and item response theory parameters using different scoring procedures [Unpublished doctoral dissertation]. Ohio University.

American Educational Research Association, American Psychological Association, \& National Council for Assessment in Education. (2014). Standards for educational and psychological testing. Washington, DC: AERA.

Boe, E. E., May, H. M., \& Boruch, R. (2002). Student task persistence in the Third International Mathematics and Science Study: A major source of achievement differences at the national, classroom, and student levels (Research Report No. 2002-TIMSS1). Center for Research and Evaluation in Social Policy, University of Pennsylvania, Philadelphia, PA. Borghans, L., \& Schils, T. (2015). The leaning tower of PISA. Working Paper. Accessed February 24. http://www. sole-jole. org/13260. pdf.

Debeer, D., Buchholz, J., Hartig, J., \& Janssen, R. (2014). Student, school, and country differences in sustained test-taking effort in the 2009 PISA reading assessment. Journal of Educational and Behavioral Statistics, 39, 502-523.

DeMars, C. E. (2000). Test stakes and item format interactions. Applied Measurement in Education, 13(1), 55-77.

Demars, C. E. (2007). Changes in rapid-guessing behavior over a series of assessments. Educational Assessment, 12(1), 23-45.

DeMars, C. E., Bashkov, B. M., \& Socha, A. B. (2013). The role of gender in test-taking motivation under low-stakes conditions. Research \& Practice in Assessment, 8, 69-82.

Deribo, T., Kroehne, U., \& Goldhammer, F. (2020). Model-based treatment of rapid 
guessing. Journal of Educational Measurement. Advanced online publication. https://doi.org/10.1111/jedm.12290

Eivers, E. (2010). PISA: Issues in implementation and interpretation. The Irish Journal of Education/Iris Eireannach an Oideachais, 38, 94-118.

Ercikan, K., \& Solano-Flores, G. (2016). Assessment and sociocultural context: A bidirectional relationship. In G. T. Brown and L. R. Harris (Eds.), Handbook of Human and Social Conditions in Assessment (pp. 490-505). Routledge.

Fox, J. P., \& Glas, C. A. (2016). Multilevel response models with covariates and multiple groups. In Handbook of Item Response Theory, Volume One (pp. 435-448). Chapman and Hall/CRC.

Freund, P.A., \& Holling, H. (2011). Who wants to take an intelligence test? Personality and achievement motivation in the context of ability testing. Personality and Individual Differences, 50(5), 723-728. http://dx.doi.org/10.1016/j.paid.2010.12.025.

Gneezy, U., List, J. A., Livingston, J. A., Sadoff, S., Qin, X., \& Xu, Y. (2017). Measuring success in education: The role of effort on the test itself (Research Report No. w24004). National Bureau of Economic Research, Cambridge, MA.

Goldhammer, F., Martens, T., Christoph, G., \& Lüdtke, O. (2016). Test-taking engagement in PIAAC (OECD Education Working Papers, No. 133). OECD Publishing.

Grisay, A., \& Monseur, C. (2007). Measuring the equivalence of item difficulty in the various versions of an international test. Studies in Educational Evaluation, 33(1), 69-86.

Haladyna, T. M, \& Downing, S. M. (1989). A taxonomy of multiple-choice item-writing rules. Applied Measurement in Education, 1, 37-50. doi:10.1207/s15324818ame0201_3

Haladyna, T. M., Rodriguez, M. C., \& Stevens, C. (2019). Are multiple-choice items too 
fat? Applied Measurement in Education, 32(4), 350-364.

Hambleton, R. K., Merenda, P. F., \& Spielberger, C. D. (2004). Adapting educational and psychological tests for cross-cultural assessment. Mahwah, NJ: Lawrence Erlbaum Associates.

Harris, L. R., \& Brown, G. T. (2016). The human and social experience of assessment: Valuing the person and context. In Brown, G. T., \& Harris, L. R. (Eds.). (2016). Handbook of human and social conditions in assessment. Routledge.

Jensen, N., Rice, A., \& Soland, J. (2018). The influence of rapidly guessed item responses on teacher value-added estimates: Implications for policy and practice. Educational Evaluation and Policy Analysis, 40(2), 267-284.

Kroehne, U., Deribo, T., \& Goldhammer, F. (2020). Rapid guessing rates across administration mode and test setting. Psychological Test and Assessment Modeling, 62(2), 147-177.

Lau, A. R., Swerdzewski, P. J., Jones, A. T., Anderson, R. D., \& Markle, R. E. (2009). Proctors matter: Strategies for increasing examinee effort on general education program assessments. The Journal of General Education, 58(3), 196-217.

Lehman, B., Jackson, G. T., \& Forsyth, C. (2019). A (mis)match analysis: Examining the alignment between test taker performance in conventional and game-based assessments. Journal of Applied Testing Technology, 20 (S1), 17-34.

Matsumoto, D., \& van de Vijver, F. J. R. (2011). Introduction to the methodological issues associated with cross-cultural research. New York, NY: Cambridge University Press.

Noble, T., Sireci, S. G., Wells, C. S., Kachchaf, R. R., Rosebery, A. S., \& Wang, Y. C. (2020). Targeted linguistic simplification of science test items for English learners. American Educational Research Journal, 57(5), 2175-2209. 
OECD (2017). OECD Handbook for Internationally Comparative Education Statistics: concepts, standards, definitions and classifications. OECD Publishing.

OECD (2019a). PISA 2018 Assessment and Analytical Framework. OECD Publishing.

OECD (2019b). PISA 2018 results: Combined executive summaries. OECD Publishing.

Osborne, J. W., \& Blanchard, M. R. (2011). Random responding from participants is a threat to the validity of social science research results. Frontiers in Psychology, 1, 220.

Penk, C., Pöhlmann, C., \& Roppelt, A. (2014). The role of test-taking motivation for students' performance in low-stakes assessments: an investigation of school-track-specific differences. Large-scale Assessments in Education, 2(1), 1-17.

Penk, C., \& Schipolowski, S. (2015). Is it all about value? Bringing back the expectancy component to the assessment of test-taking motivation. Learning and Individual Differences, 42, 27-35.

Pintrich, P. R., \& Schunk, D. H. (2002). Motivation in education: Theory, Research, and Applications (2 $2^{\text {nd }}$ ed.). Columbus, OH: Merrill Prentice Hall.

Pizmony-Levy, O., Harvey, J., Schmidt, W. H., Noonan, R., Engal, L., Feuer, M. J., ...TorneyPurta, J. (2014). On the merits of, and myths about, international assessments. Quality Assurance in Education, 22, 319 - 338.

Rios, J. A. (2021a). Is differential noneffortful responding associated with type I error in measurement invariance testing? Educational and Psychological Measurement, 81(5), 957-979.

Rios, J. A. (2021b). Assessing the accuracy of parameter estimates in the presence of rapid guessing misclassifications. Educational and Psychological Measurement. Advanced online publication. doi: 00131644211003640. 
Rios, J. A., \& Deng, J. (2021). Does the choice of response time threshold procedure substantially affect inferences concerning the identification and exclusion of rapid guessing responses? A meta-analysis. Large-scale Assessments in Education, 9(1), 1-25.

Rios, J. A., \& Guo, H. (2020). Can culture be a salient predictor of test-taking engagement? An analysis of differential noneffortful responding on an international college-level assessment of critical thinking. Applied Measurement in Education, 33(4), 263-279.

Rios, J. A., Guo, H., Mao, L., \& Liu, O. L. (2017). Evaluating the impact of careless responding on aggregated-scores: To filter unmotivated examinees or not?. International Journal of Testing, 17(1), 74-104.

Rios, J. A., \& Liu, O. L. (2017). Online proctored versus unproctored low-stakes internet test administration: Is there differential test-taking behavior and performance? American Journal of Distance Education, 31(4), 226-241.

Rios, J. A., Liu, O. L., \& Bridgeman, B. (2014). Identifying low-effort examinees on student learning outcomes assessment: A comparison of two approaches. New Directions for Institutional Research, 2014(161), 69-82.

Rios, J. A., \& Soland, J. (2021). Parameter estimation accuracy of the Effort-Moderated IRT model under multiple assumption violations. Educational and Psychological Measurement, 81(3), 569-594. doi: 10.1177/0013164420949896

Russell, M., \& Moncaleano, S. (2019). Examining the use and construct fidelity of technologyenhanced items employed by K-12 testing programs. Educational Assessment, 24(4), 286-304.

Schneider, M. C., Huff, K. L., Egan, K. L., Gaines, M. L., \& Ferrara, S. (2013). Relationships 
among item cognitive complexity, contextual demands, and item difficulty: Implications for achievement-level descriptors. Educational Assessment, 18(2), 99-121.

Setzer, J. C., Wise, S. L., van den Heuvel, J. R., \& Ling, G. (2013). An investigation of examinee test-taking effort on a large-scale assessment. Applied Measurement in Education, 26(1), 34-49.

Silm, G., Pedaste, M., \& Täht, K. (2020). The relationship between performance and test-taking effort when measured with self-report or time-based instruments: A meta-analytic review. Educational Research Review, 31, 100335.

Smith, J. K., Given, L. M., Julien, H., Ouellette, D., \& DeLong, K. (2013). Information literacy proficiency: Assessing the gap in high school students' readiness for undergraduate academic work. Library \& Information Science Research, 35(2), 88-96.

Soland, J. (2018a). Are achievement gap estimates biased by differential student test effort? Putting an important policy metric to the test. Teachers College Record, 120(12), 1-26.

Soland, J. (2018b). The achievement gap or the engagement gap? Investigating the sensitivity of gaps estimates to test motivation. Applied Measurement in Education, 31(4), 312-323.

Soland, J., Jensen, N., Keys, T. D., Bi, S. Z., \& Wolk, E. (2019). Are test and academic disengagement related? Implications for measurement and practice. Educational Assessment, 24(2), 119-134.

Suárez-Orozco, C., Rhodes, J., \& Milburn, M. (2009). Unraveling the immigrant paradox: Academic engagement and disengagement among recently arrived immigrant youth. Youth \& Society, 41(2), 151-185.

Webb, N. (1997). Criteria for alignment of expectations and assessments in mathematics and science education. National Institute for Science Education. 
Wigfield, A., \& Eccles, J. S. (2000). Expectancy-value theory of achievement motivation. Contemporary Educational Psychology, 25, 68-81.

Wise, S. L. (2006). An investigation of the differential effort received by items on a low-stakes computer-based test. Applied Measurement in Education, 19(2), 95-114.

Wise, S. L. (2015). Effort analysis: Individual score validation of achievement test data. Applied Measurement in Education, 28(3), 237-252.

Wise, S. L. (2017). Rapid-guessing behavior: Its identification, interpretation, and implications. Educational Measurement: Issues and Practice, 36(4), 52-61.

Wise, S. L. (2019). Controlling construct-irrelevant factors through computer-based testing: Disengagement, anxiety, \& cheating. Education Inquiry, 10(1), 21-33.

Wise, S. L., Bhola, D. S., \& Yang, S. T. (2006). Taking the time to improve the validity of lowstakes tests: The effort-monitoring CBT. Educational Measurement: Issues and Practice, 25(2), 21-30.

Wise, S. L., \& DeMars, C. E. (2005). Examinee motivation in low-stakes assessment: Problems and potential solutions. Educational Assessment, 10, 1-18.

Wise, S. L., \& DeMars, C. E. (2010). Examinee noneffort and the validity of program assessment results. Educational Assessment, 15, 27-41.

Wise, S. L., \& Kingsbury, G. G. (2016). Modeling student test-taking motivation in the context of an adaptive achievement test. Journal of Educational Measurement, 53(1), 86-105.

Wise, S., \& Kuhfeld, M. (2021). A method for identifying partial test-taking engagement. Applied Measurement in Education, 1-12.

Wise, S. L., Kuhfeld, M. R., \& Soland, J. (2019). The effects of effort monitoring with proctor 
notification on test-taking engagement, test performance, and validity. Applied Measurement in Education, 32(2), 183-192.

Wise, S. L., \& Ma, L. (2012, April). Setting response time thresholds for a CAT item pool: The normative threshold method. Paper presented at the annual meeting of the National Council on Measurement in Education, Vancouver, Canada.

Wise, S. L., Pastor, D. A., \& Kong, X. J. (2009). Correlates of rapid-guessing behavior in lowstakes testing: Implications for test development and measurement practice. Applied Measurement in Education, 22(2), 185-205.

Wise, S. L., Soland, J., \& Bo, Y. (2020). The (Non) impact of differential test taker engagement on aggregated scores. International Journal of Testing, 20(1), 57-77.

Wise, S. L., Soland, J., \& Dupray, L. M. (2021). The impact of technology-enhanced items on test-taker disengagement. Journal of Applied Testing Technology, 22(1), 28-36.

Wolf, L. F., Smith, J. K., \& Birnbaum, M. E. (1995). Consequence of performance, test, motivation, and mentally taxing items. Applied Measurement in Education, 8(4), 341351.

Zamarro, G., Hitt, C., \& Mendez, I. (2019). When students don’t care: Reexamining international differences in achievement and student effort. Journal of Human Capital, 13(4), 519-552. 
Table 1

Examinee- and Country-level Predictors of Rapid Guessing

\begin{tabular}{|c|c|c|c|}
\hline Predictor & Estimate & S.E. & P-Value \\
\hline \multicolumn{4}{|l|}{ Country-level predictors } \\
\hline Mean Score & -0.002 & 0.001 & $0.000 *$ \\
\hline Sex Gap & 0.002 & 0.003 & 0.270 \\
\hline OECD & -0.082 & 0.064 & 0.160 \\
\hline Americas & -0.112 & 0.073 & 0.070 \\
\hline Asia & 0.165 & 0.069 & $0.020 *$ \\
\hline Middle East \& Africa & 0.258 & 0.111 & $0.010 *$ \\
\hline \multicolumn{4}{|c|}{ Examinee-level predictors (country-level means) } \\
\hline SES & -0.062 & 0.004 & $0.000 *$ \\
\hline Immigrant & 0.020 & 0.024 & 0.160 \\
\hline Sex & 0.110 & 0.009 & $0.000 *$ \\
\hline Read Comp. & -0.055 & 0.004 & $0.000 *$ \\
\hline Fear Failure & -0.003 & 0.003 & 0.200 \\
\hline Self-efficacy & 0.004 & 0.004 & 0.180 \\
\hline Motivation & -0.055 & 0.004 & $0.000 *$ \\
\hline Home Language & 0.118 & 0.018 & $0.000 *$ \\
\hline \multicolumn{4}{|l|}{ Within-country variances } \\
\hline SES & 0.001 & 0.000 & $0.000 *$ \\
\hline Immigrant & 0.013 & 0.006 & $0.000 *$ \\
\hline Sex & 0.005 & 0.001 & $0.000 *$ \\
\hline Read Comprehension & 0.001 & 0.000 & $0.000 *$ \\
\hline Fear Failure & 0.001 & 0.000 & $0.000 *$ \\
\hline Self-efficacy & 0.001 & 0.000 & $0.000^{*}$ \\
\hline Motivation & 0.001 & 0.000 & $0.000 *$ \\
\hline Home Language & 0.017 & 0.003 & $0.000 *$ \\
\hline
\end{tabular}

Note. ${ }^{*}$ means the parameter estimate is significant at the .05 level. 
Table 2

Examinee- and Item-level Predictors of Rapid Guessing

\begin{tabular}{lccc}
\hline Predictor & Estimate & S.E. & P-Value \\
\hline Examinee-level predictors & & & \\
Sex & 0.105 & 0.006 & $0.000^{*}$ \\
Home Language & 0.078 & 0.005 & $0.000^{*}$ \\
Immigrant & 0.036 & 0.007 & $0.000^{*}$ \\
SES & -0.075 & 0.002 & $0.000^{*}$ \\
Self-efficacy & 0.003 & 0.002 & 0.090 \\
Fear Failure & -0.005 & 0.002 & $0.020^{*}$ \\
Reading Comprehension & -0.054 & 0.002 & $0.000^{*}$ \\
Motivation & -0.043 & 0.002 & $0.000^{*}$ \\
& & & \\
Item-level predictors & & & \\
Interactive Stimulus & -0.238 & 0.082 & $0.000^{*}$ \\
Sequence in Item Cluster & -0.005 & 0.006 & 0.240 \\
Complex Multiple-Choice Item & 0.097 & 0.092 & 0.130 \\
Open Response Item & 0.053 & 0.096 & 0.270 \\
Cognitive Demand Medium & 0.127 & 0.081 & 0.050 \\
Cognitive Demand High & -0.083 & 0.149 & 0.240 \\
Item Difficulty & 0.000 & 0.002 & 0.420 \\
\hline
\end{tabular}

Note. * means the parameter estimate is significant at the .05 level. 
Figure 1

Average Proportion of RG Responses per Examinee by Country

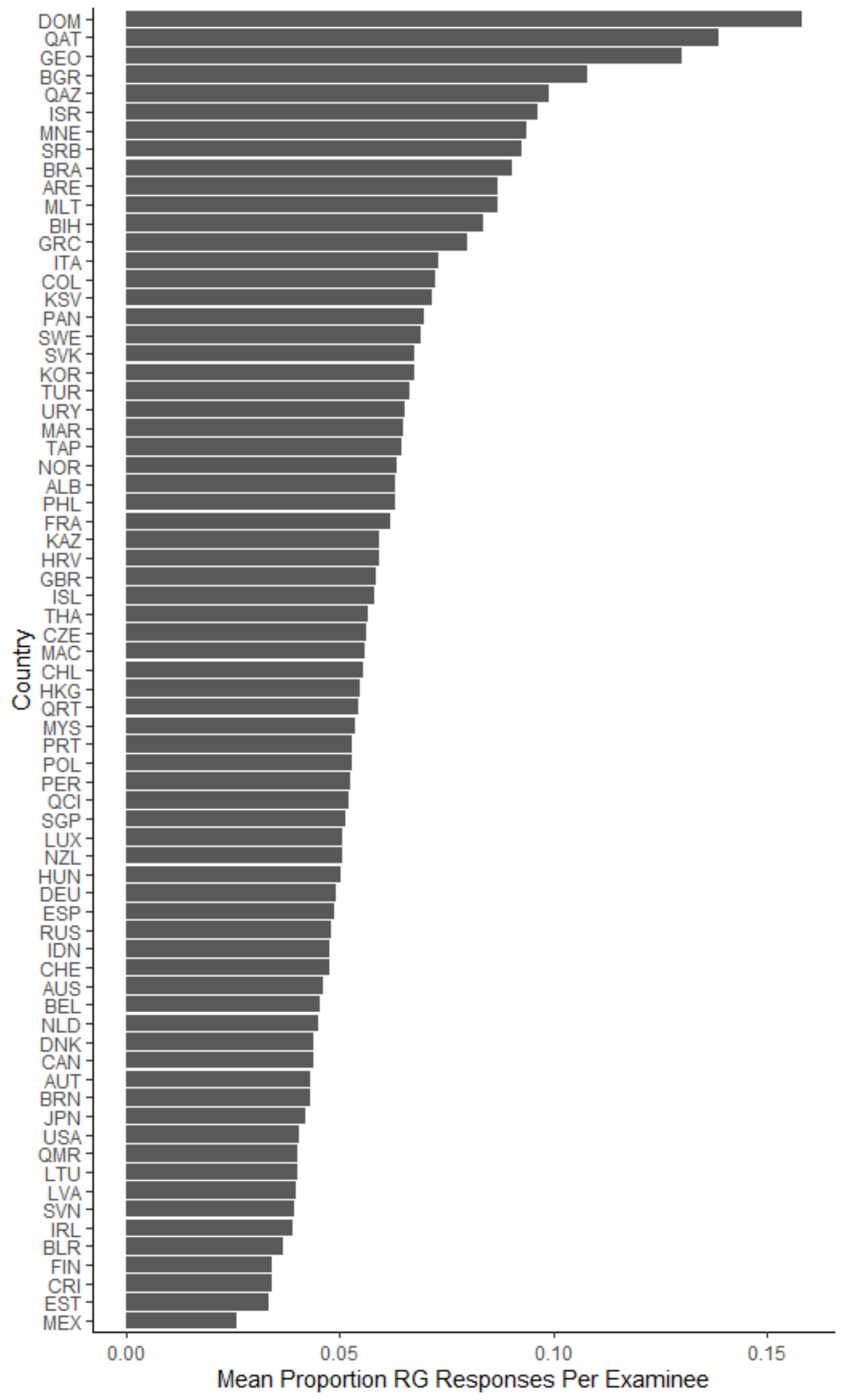

